\title{
Effects of TeV Scale Gravity on $e^{+} e^{-} \rightarrow W^{+} W^{-}$
}

\author{
P. Poulose屯 \\ Institute of Theoretical Physics E, RWTH Aachen, \\ D-52056 Aachen, Germany
}

\begin{abstract}
We study the process $e^{+} e^{-} \rightarrow W^{+} W^{-}$to probe low-scale gravity at high energy linear colliders. A characteristic signature of extra-dimensions models is the forward-backward asymmetry due to the interference of spin2 graviton and spin-1 SM gauge boson exchange terms, even when rightpolarised electron beam is used. Our analysis shows that larger than 5\% asymmetry is possible at a linear collider with $\sqrt{s}=500$ (800) $\mathrm{GeV}$ if the mass scale, $M_{S}$ is smaller than $2.7(4.5) \mathrm{TeV}$. $W^{-}$polarisation factors measured with a few percent accuracy will also be able to put similar limits on $M_{S}$.
\end{abstract}

\section{Introduction}

In this paper we study the process $e^{+} e^{-} \rightarrow W^{+} W^{-}$in the presence of low-scale gravity with extra spatial dimensions. In such a large extra-dimensions scenario gravity is allowed to propagate in $D=4+n$ dimensions, while SM particles confine to a sub-space of 4-dimensions. This idea was proposed by Arkani-Hamed, Dimopoulos and Dvali [1], and the phenomenological consequences at colliders have been looked at in various processes in detail by many authors [2]. The main idea is to have a universally fundamental-scale, $M_{S}$, of $\mathrm{TeV}$ range where all interactions including gravity are comparable in strength. This is contrary to the traditional belief that gravity becomes comparable in stregth to the other interactions only at Planck mass, $M_{P} \sim 10^{19} \mathrm{GeV}$. This lowering of the scale is possible if we consider $n$ spatial dimensions in addition to the usual $3+1$ space-time manifold. These additional dimensions are compactified to a radius $R$. Relation between the two mass scales, the size of the extra dimensions and the number of extra dimensions is obtained by demanding that for distances larger than $R$ gravitational potential behaves like the Newtonian $1 / r$. Thus we get the relation[1]

$$
M_{P}^{2}=8 \pi R^{n} M_{S}^{n+2} .
$$

With an $M_{S} \sim \mathrm{TeV}, n=1$ requires $R$ to be of the order of $10^{11}$ meters. This is ruled out, since Newtonian gravity is tested to be correct up to about millimeter level. $n=2$ or larger is allowed by this criterion. Thus gravity can propagate in $4+n$ dimensions as long as the extra $n$ dimensions are smaller than 1 $\mathrm{mm}$. Since Standard Model (SM) interactions are tested up to sub-fermi level, we

\footnotetext{
${ }^{1}$ poulos@physik.rwth-aachen.de
} 
require to confine these particles to a 4 dimensional sub-space. ${ }^{2}$ Massless gravitons propagating in the bulk is seen from the 4 dimensional sub-manifold as massive Kaluza-Klein (KK) modes with spin-0, spin-1 and spin-2. Mass spectrum can be treated to be continuous owing to the fact that mass splitting is of the order of $1 / R$, which is about $10^{-4} \mathrm{eV}$ in the case of $n=2$, and of the order of $\mathrm{MeV}$ in the case of $n=6$. These KK modes couple to matter and gauge particles through the energy-momentum tensor and its trace. The new interactions influence collider experiments in two ways. One through the production of real KK modes, and two through the exchange of virtual KK modes in various processes. For a review on the effects of this idea in linear colliders like TESLA, see [4].

In the following we will consider the process $e^{+} e^{-} \rightarrow W^{+} W^{-}$to study the effect of low-scale gravity at the proposed linear colliders to run at $500 \mathrm{GeV}$ and above. Earlier studies of this process [7, 8] have discussed possible limits on $M_{S}$ obtainable measuring, mainly, deviation of the total cross section from its SM value. We will discuss, in addition to the total cross section, other observables like the forwardbackward asymmetry and polarisation fraction of the $W$ 's. In a linear collider it is possible to use polarised beams. We will discuss the benifits of beam polarisation in the present case.

In section 2 we discuss the process and give the expressions for matrix elements. Section 3 describes different observables that are relevant at a high energy linear collider. Conclusions will be given in section 4 .

\section{The process}

The process $e^{+} e^{-} \rightarrow W^{+} W^{-}$has the standard interactions with the exchange of photon and $Z$-boson in the $s$-channel and a $t$-channel with $\nu$ exchange. In the case of large extra dimensions, there are additional $s$-channels with spin- $2 \mathrm{KK}$ excitations of bulk gravitons. Spin-1 modes do not couple to the SM particles, and spin-0 contributions are proportional to the mass of the fermion invovled. We treat electron as massless, and so neglect spin-0 exchange contribution.

The Feynman diagrams of different channels are given in Fig. 11.

Relevant Feynman rules are derived by Han, Lykken and Zhang [5], and also by Guidice, et al [6] in a linearised-gravity aproximation. There is an infinite tower of massive KK excitations all having a universal coupling to the SM particles. Han, Lykken and Zhang [5] have derived the propagator factor after integrating over the spectrum, cutting it at the scale $M_{S}$. Following them we get KK exchange contribution to the matrix element given by

$$
\begin{aligned}
\mathcal{M}_{G}=-2 \pi C_{4} & \left\{\left[2\left(k_{1} \cdot q_{2}-k_{1} \cdot q_{1}\right)\left(\epsilon_{1} \cdot \epsilon_{2}\right)+2\left(k_{1} \cdot \epsilon_{1}\right)\left(q_{1} \cdot \epsilon_{2}\right)-2\left(k_{1} \cdot \epsilon_{2}\right)\left(q_{2} \cdot \epsilon_{1}\right)\right]\left(\bar{v} q_{1} u\right)\right. \\
& +\left[-2\left(k_{1} \cdot q_{2}\right)\left(q_{1} \cdot \epsilon_{2}\right)+s\left(k_{1} \cdot \epsilon_{2}\right)\right]\left(\bar{v} \phi_{1} u\right)
\end{aligned}
$$

\footnotetext{
${ }^{2}$ Alternative scenarios with SM particles allowed to move in the bulk are discussed in the literature. There is also the scenario proposed by Randall and Sundrum[3] with non-factorizable metric. We will not consider any of these cases here.
} 

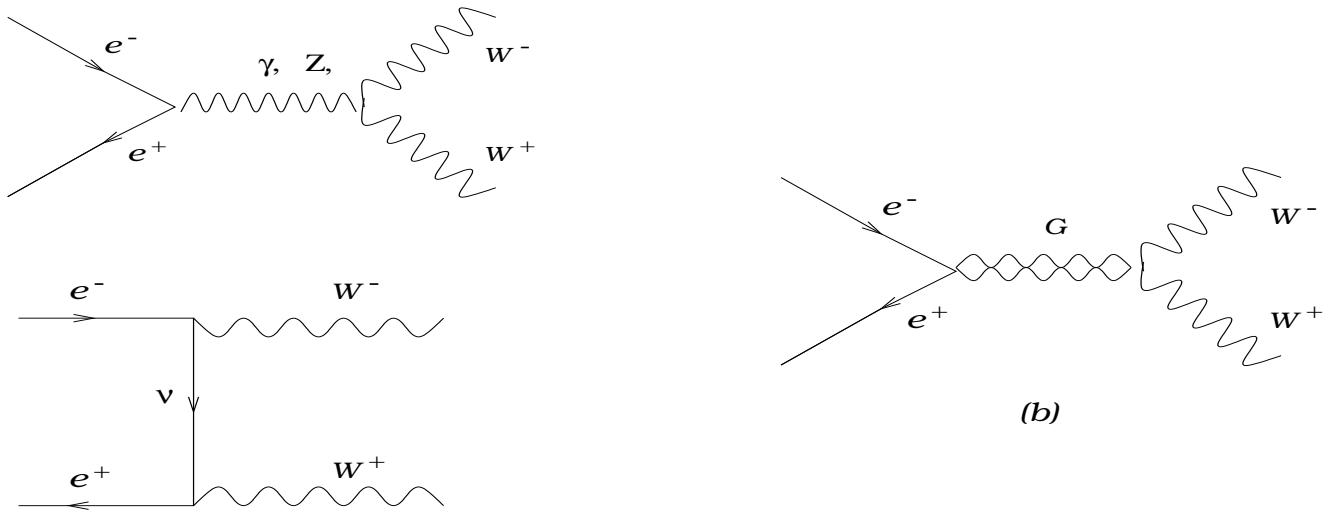

(a)

Figure 1: Feynman diagramms of the process $e^{+} e^{-} \rightarrow W^{+} W^{-}$. (a) SM channels and (b) Spin-2 KK exchange channel.

$$
\left.+\left[-2\left(k_{1} \cdot q_{1}\right)\left(q_{2} . \epsilon_{1}\right)+s\left(k_{1} \cdot \epsilon_{1}\right)\right]\left(\bar{v} ф_{2} u\right)\right\}
$$

Here $k_{1}$ is the momentum of initial $e^{-}$, and $u$ and $v$ are the spinors corresponding to $e^{-}$and $e^{+} . q_{1}$ and $q_{2}$ are the momenta, and $\epsilon_{1}$ and $\epsilon_{2}$ the polarisation vectors of $W^{-}$and $W^{+}$respectively. $\sqrt{s}$ is the centre of masss energy of the collider, and $C_{4}$ is the factor corresponding to KK propagators after integrating over all the modes.

The propagator factor in the limit of $M_{S}^{2} \gg s$ is given by

$$
\begin{array}{rlr}
C_{4} & =-\frac{\log \left(M_{S}^{2} / s\right)}{M_{S}^{4}} & (n=2) \\
& =-\frac{2}{(n-2) M_{S}^{4}} \quad(n>2)
\end{array}
$$

Here $n$ is the number of large extra dimensions. In the case of high energy colliders expected to operate at energies of $500 \mathrm{GeV}$ through 1-2 TeV, $M_{S}^{2} \gg s$ may not be a good approximation. In that case, nonresonant part of the propagator factors, neglecting the narrow width of each KK mode, as given by [5] are,

$$
\begin{aligned}
C_{4} & =\left(\frac{s}{M_{S}^{2}}\right)^{n / 2-1} \frac{2 I_{n}}{M_{S}^{4}} \\
I_{n} & = \begin{cases}-\sum_{k=1}^{n / 2-1} \frac{1}{2 k}\left(\frac{M_{S}}{\sqrt{s}}\right)^{2 k}-\frac{1}{2} \log \left(\frac{M_{S}^{2}}{s}-1\right) & (\text { even } n) \\
-\sum_{k=1}^{(n-1) / 2} \frac{1}{2 k-1}\left(\frac{M_{S}}{\sqrt{s}}\right)^{2 k-1}+\frac{1}{2} \log \left(\frac{M_{S}+\sqrt{s}}{M_{S}-\sqrt{s}}\right) & (\text { odd } n)\end{cases}
\end{aligned}
$$

Apart from these non-resonant contributions, there is also a resonant contribution to the propagator factor, which adds an imaginary part to the matrix element 
[5]. In the case of $e^{+} e^{-} \rightarrow W^{+} W^{-}$, since the SM matrix elements are real, there is no contribution from this part in the interference term. Purely gravity mediated contribution will be of order $s^{4} / M_{S}^{8}$, and is negligible unless $\sqrt{s}$ is very close to $M_{S}$.

Explicit expressions for different helicity amplitudes are given in [7]. We consider full propagator factor including its dependence on the number of extra dimensions, as given above. Thus to compare with the expressions given in [7] the factor $\lambda \frac{4}{M_{S}^{4}}$ should be replaced by $-2 \pi C_{4}$.

In the next section we discuss various observables that could be used at a high energy linear collider.

\section{Observables}

Possible limits on $M_{S}$ that could be obtained at future linear colliders (LC) from considering the deviation of total cross section from the SM expectation were obtained in earlier studies [7, 8]. Limits obtained depend on the aproximations used to obtain KK-propagator factor. Our main aim in this report is to identify possible signatures which characterize the spin-2 nature of the graviton exchange. The angular distribution of the $W$ 's and the forward-backward asymmetry, as we will see, are good candidates in this respect.

\subsection{Unpolarised $W$ 's}

We first consider typical limit on $M_{S}$ that one could obtain at an LC. In Table 1 we give limits on $M_{S}$ that would correspond to a $2 \%$ deviation in the SM cross section at $\mathrm{LC}$ with c.m. energies of $500 \mathrm{GeV}$ and $800 \mathrm{GeV}$. We assume $100 \%$ efficiency, and have not considered any experimental cuts. More realisitic considerations will change the limits somewhat. Large beam polarisations can be achieved in linear colliders. Using a right-polarised electron beam switches off the $\nu$ exchange contribution, and hence improves the sensitivity to graviton exchange contribution. At a $500 \mathrm{GeV}$ collider, while using unpolarised beams or left-polarised electron beam with unpolarised positron beam could reach $M_{S}$ values from about $1.3 \mathrm{TeV}$ (for $n=2$ ) up to about $1.8 \mathrm{TeV}$ (for $n=6$ ), right-polarised electron beam with unpolarised positron beam could reach up to about $2.7 \mathrm{TeV}$.

Next we consider angular distribution of the $W$ 's. Fig.2 shows angular distributions of $W$ 's for a typical value of $M_{S}=2 \mathrm{TeV}$. In the SM case there is a forward-backward asymmetry when unpolarised or left-polarised electron beam is considered. This is due to the $\nu$ exchange contribution. With right-handed electron polarisation there is no $\nu$ exchange contirbution and hence the SM distribution is symmetric. When graviton exchange contribution is included, the intereference of $J=2$ and $J=1$ gives rise to an asymmetric distribution even when the electron beam is right-polarised. This was also noticed by the authors of [7]. In the following we quantify this forward-backward asymmetry. 


\begin{tabular}{|c|c|c|c|c|c|c|}
\hline \multirow[b]{2}{*}{$\sqrt{s}$} & \multirow[b]{2}{*}{$e^{-}$beam pol } & \multicolumn{5}{|c|}{ Limit on $M_{S}$ in $\mathrm{TeV}$} \\
\hline & & $n=2$ & $n=3$ & $n=4$ & $n=5$ & $n=6$ \\
\hline \multirow[t]{2}{*}{$500 \mathrm{GeV}$} & Unpol / $e_{L}^{-}$ & 1.8 & 1.7 & 1.5 & 1.4 & 1.3 \\
\hline & $e_{R}^{-}$ & 2.7 & 2.2 & 1.9 & 1.7 & 1.6 \\
\hline \multirow[t]{2}{*}{$800 \mathrm{GeV}$} & Unpol / $e_{L}^{-}$ & 2.6 & 2.5 & 2.3 & 2.1 & 2.0 \\
\hline & $e_{R}^{-}$ & 4.5 & 3.6 & 3.2 & 2.9 & 2.7 \\
\hline
\end{tabular}

Table 1: $M_{S}$ values corresponding to $2 \%$ deviation in the SM cross section at LC with $\sqrt{s}=500 \mathrm{GeV}$ and $\sqrt{s}=800 \mathrm{GeV}$ for different beam polarisations. $n$ is the number of extra dimensions.
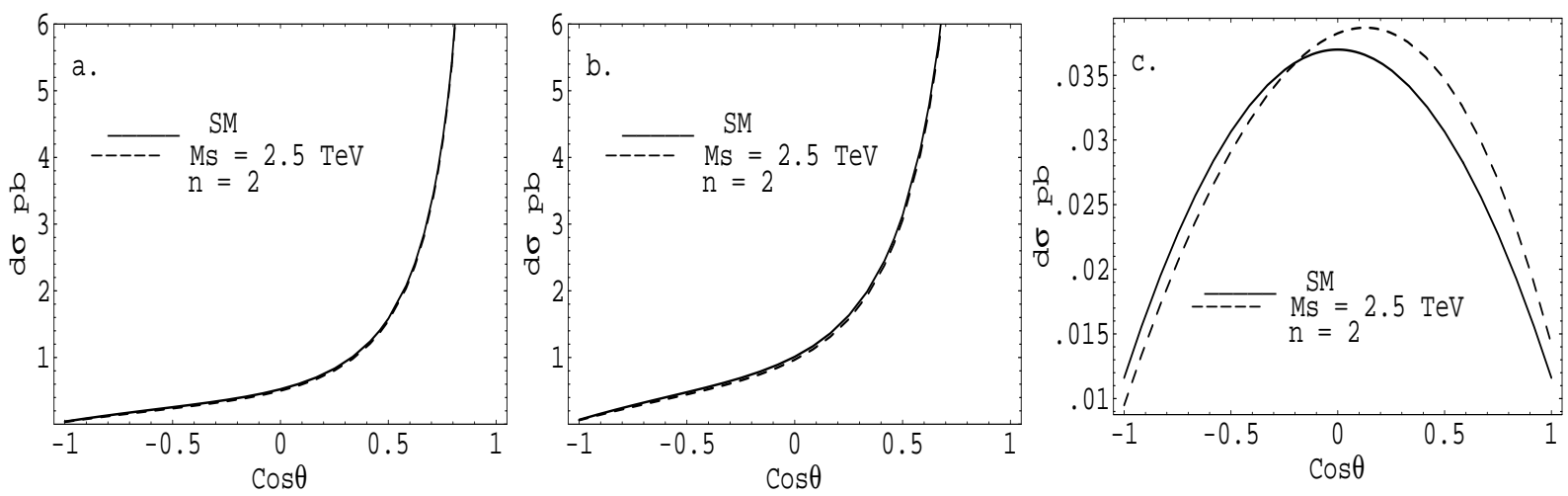

Figure 2: Angular distribution of $W$ 's at an LC with $\sqrt{s}=500 \mathrm{GeV}$. Solid curves correspond to SM, and dashed curves correspond to SM+Gravity with $M_{S}=2.5$ TeV and $n=2$. (a), (b) and (c) correspond to unpolarised, left-polarised and rightpolarised electron beams respectively. Positron beam is considered unpolarised.

The forward-backward asymmetry as a function of $M_{S}$ is plotted in Fig. 3 . With $M_{S}=2.5 \mathrm{TeV}$ and $n=2$ asymmetry can be as large as $8 \%$ at $500 \mathrm{GeV}$. The asymmetry tends to vanish as $\sqrt{s}$ aproaches $M_{S}$. This is because, then the purely gravity mediated contribution dominates over the interference of the SM and gravity mediated contribution. With $n=2$ maximum asymmetry is around $15 \%$ corresponding to $M_{S}$ value of $1.7(2.7) \mathrm{TeV}$ at $\sqrt{s}=500(800) \mathrm{GeV}$. For the asymmetry to be at the level of $5 \%$ or more, $M_{S}$ values should be less than 2.8 (1.8) $\mathrm{TeV}$ for $n=2$ (6) at a $500 \mathrm{GeV} \mathrm{LC}$, and 4.5 (2.9) TeV at an $800 \mathrm{GeV} \mathrm{LC.}$

\subsection{Polarised $W^{\prime}$ 's}

We now consider production of polarised $W^{\prime}$ s. $W$ polarisation can be measured by studying its decay distributions. We study the polarisation fractions, $f_{0, \pm}$ of the $W$ 's produced. They enter in the expression for angular distribution of the 

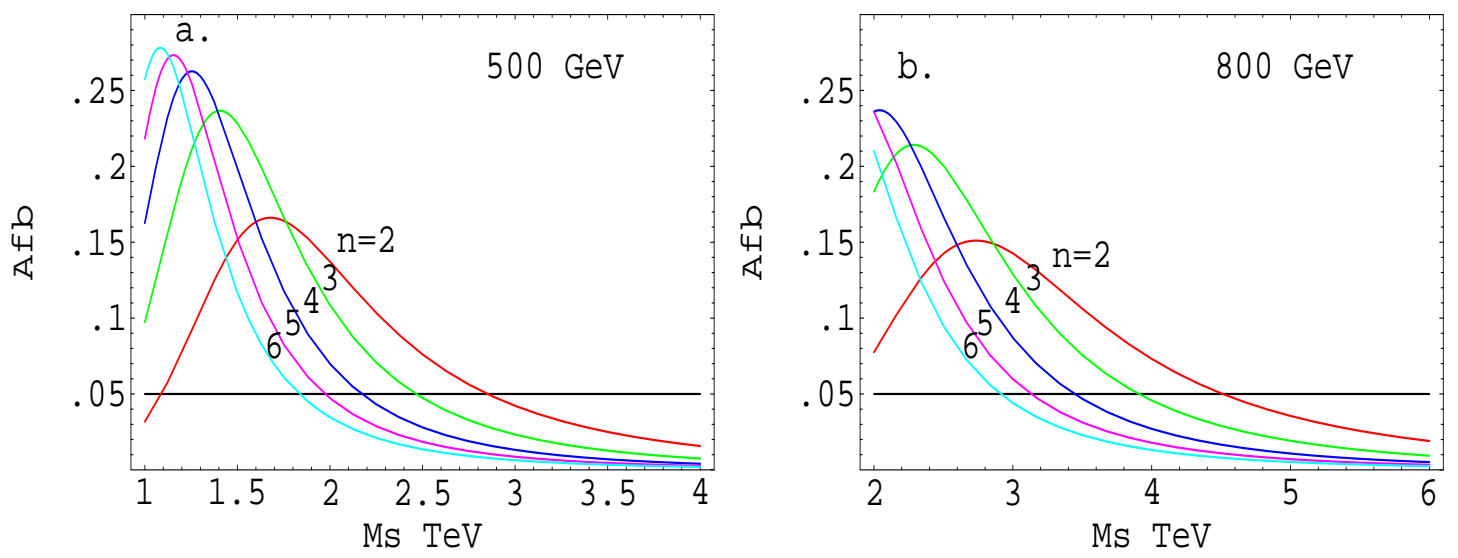

Figure 3: Forward-backward asymmetry with right-polarised electron beam. (a) $\sqrt{s}=500 \mathrm{GeV}$ and (b) $\sqrt{s}=800 \mathrm{GeV} . n$ is the number of extra dimensions.

secondary lepton in the rest frame of the $W$ in the following way.

$$
\frac{1}{\sigma} \frac{d \sigma}{d \cos \theta^{*}}=\frac{3}{4} f_{0} \sin ^{2} \theta^{*}+\frac{3}{8} f_{+}\left(1-\cos \theta^{*}\right)^{2}+\frac{3}{8} f_{-}\left(1+\cos \theta^{*}\right)^{2},
$$

Here $\theta^{*}$ is the polar angle of the lepton in the rest frame of the $W$ with $z$ axis along the boost direction. $f_{0}$ gives the fractional cross section of the longitudinal $W^{-}$, while $f_{ \pm}$give that of the right-/left-circularly polarised $W^{-}$'s. LEP2 has been able to measure the longitudinal fraction, $f_{0}$ with an accuracy of $5 \%$ [9].

Table 2 gives different polarisation fractions without and with contribution due the graviton-KK exchange at an LC with right-handed electron beam.

\begin{tabular}{c|c|c|c|c|c}
\hline$\sqrt{s}$ & $M_{S}$ & $f_{0}^{S M}$ & $f_{0}^{G}$ & $f_{ \pm}^{S M}$ & $f_{ \pm}^{G}$ \\
\hline $500 \mathrm{GeV}$ & $2.5 \mathrm{TeV}$ & 0.860 & 0.834 & 0.070 & 0.083 \\
\hline $800 \mathrm{GeV}$ & $4 \mathrm{TeV}$ & 0.932 & 0.897 & 0.034 & 0.052 \\
\hline
\end{tabular}

Table 2: Fractional polarisations of the $W$ 's with right-handed electron beam. $f_{0, \pm}^{S M}$ 's are SM values, while $f_{0, \pm}^{G}$ 's those with graviton exchange included. Number of extra dimensions is taken to be $n=2$. Note that $f_{+}=f_{-}$.

Although the cross section with right-handed electron polarisation is only about 57.1 (18.7) fb at $\sqrt{s}=500(800) \mathrm{GeV}$, a collider with luminosity of the order of $100 \mathrm{fb}^{-1}$ will produce several thousand events. This would allow a determination of $f_{0}$ with an error of 1-2\%, sufficient to probe the effects of grativon exchange indicated in Table 2 . 


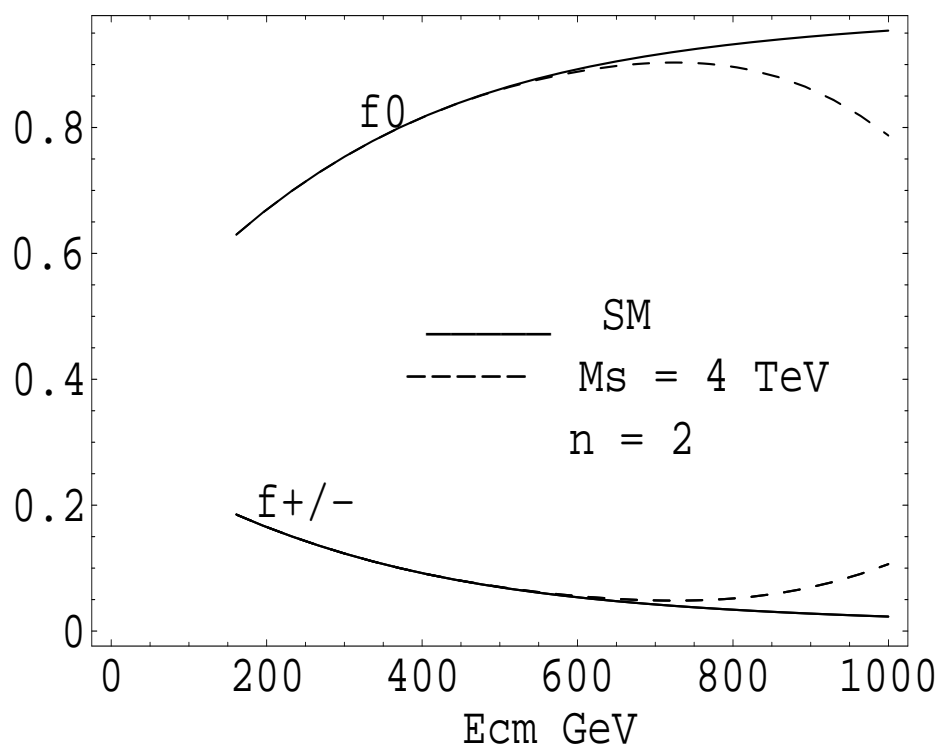

Figure 4: Polarisation fractions of $W^{-}$'s with right-handed electron beam. Solid curves correspond to SM values, while dashed curves correspond to values with graviton exchange with $M_{S}=4 \mathrm{TeV}$ and number of extra dimensions, $n=2$ included.

\section{Conclusion}

We have considered the process $e^{+} e^{-} \rightarrow W^{+} W^{-}$to study the effect of a typical low energy gravity model suggested by Arkani-Hamed, Dimopoulos and Dvali [1]. In such a model, in addition to the usual SM contribution, there is a contribution due to the spin-2 KK exchange in the $s$-channel. Earlier studies have obtained limits on the mass scale $M_{S}$, at which gravity becomes comparable in strength to the other interactions, in $4+n$ dimensions [7, 8]. These limits are obtained considering deviation of the total cross section from its SM value. In this paper we have considered observables which help distinguish the spin-2 nature of the interaction. A forward-backward asymmetry in the case of right-polarised electron beam is one significant observable in this regard. We have quantified the asymmetry, and considered reach of $M_{S}$ by looking at this asymmetry at a linear collider running at $\sqrt{s}=500 \mathrm{GeV}$ and $800 \mathrm{GeV}$. We also looked at the polarisation fractions of the $W$ 's produced. Here again, we reach the conclusion that right-polarised electron beam is advantageous. Our analysis shows that an $M_{S}$ of about $2.5 \mathrm{TeV}$ could produce detectable effects at a linear collider running at $500 \mathrm{GeV}$ centre of mass energy. This is improved to about $4 \mathrm{TeV}$ for a centre of mass energy of $800 \mathrm{GeV}$. The effects are qualitatively different from other new physics scenarios, e.g., strong $W W$ interactions [10] or extra gauge boson models [11], since these affect the $J=1$ partial waves, while graviton exchange is specifically a $J=2$ phenomenon. 


\section{Acknowledgement}

I would like to thank Prof. L.M.Sehgal for suggesting this problem, and for many discussions. I also wish to thank the Humboldt Foundation for a Postdoctoral Fellowship, and the Institute of Theoretical Physics E, RWTH Aachen for the hospitality provided during this work.

\section{References}

[1] N. Arkani-Hamed, S. Dimopoulos and G. R. Dvali, Phys. Lett. B429 (1998) 263; Phys. Rev. D59 (1999) 086004; I. Antoniadis, N. Arkani-Hamed, S. Dimopoulos and G.R. Dvali, Phys. Lett. B436 (1998) 257.

[2] For reviews see: Yuri A. Kubyshin, hep-ph/0111027; K. Sridhar, Int. J. Mod. Phys. A15 (2000) 2397 (hep-ph/0004053); T. G. Rizzo, hep-ph/9910255.

[3] L. Randall and R. Sundrum, Phys. Rev. Lett. 83 (1999) 4690; Phys. Rev. Lett. 83 (1999) 3370.

[4] R.D. Heuer, D. Miller, F. Richard, P. Zerwas, TESLA Technical Design Report: Part III, DESY-2001-011 (hep-ph/0106315); G. Pasztor, T. G. Rizzo, Snowmass 2001, hep-ph/0112054

[5] T. Han, J. D. Lykken and Ren-Jie Zhang, Phys. Rev. D59 (1999) 105006.

[6] G. F. Guidice, et al., Nucl. Phys. B544 (1999) 3.

[7] K. Agashe and N.G. Deshpande, Phys. Lett. B456 (1999) 60.

[8] C. Balázs, et al, Phys. Rev. Lett 83 (1999) 2112.

[9] L3 Collaboration, Phys. Lett. B74 (2000) 194; L3 Note 2636

[10] P. Poulose S. D. Rindani and L. M. Sehgal, Phys. Lett. B525 (2002) 71, and references therein.

[11] S. Godfrey, Phys. Rev. D51 (1995) 1402; Dong-Won Jung, K. Y. Lee, H.S. Song, C. Yu, hep-ph/9905353. See also Ref. [⿴囗十. 